\title{
Descriptive Analysis of Factors Associated with COVID-19 (U07.1, U07.2), Viral Pneumonia (J12.8 and J12.9) and other Types of Admission Diagnosis
}

\author{
Michael Mncedisi Willie* \\ Policy Research and Monitoring, Council for Medical Schemes, Pretoria, South Africa
}

\begin{abstract}
Background: Various studies have drawn similarities between respiratory conditions such as pneumonia and COVID-19. Respiratory disease (e.g., viral pneumonia J12.8) or signs or symptoms of respiratory disease (e.g., shortness of breath R06.0, cough R05) a respiratory disease (e.g., viral pneumonia J12.8) are the most notable symptoms that are associated with COVID-19. Pneumonia is also among the top five most prevalent reasons for admissions in medical schemes and is one of the expensive conditions to treat. This further accounts for a significant portion of hospitalisation expenditure.
\end{abstract}

Objectives: The primary objective of this study was to study the characteristics of medical scheme patient's viral pneumonia as a primary ICD-10 diagnosis and ICD-10 secondary diagnosis that were admitted into private hospitals.

Methods: The study design was a descriptive analysis of medical schemes admissions data that occurred in 2020. The review period was claims received between March to August 2020. The inclusion criteria were patients viral pneumonia primary ICD-10 code. Patients with a secondary ICD-10 code who had a COVID-19 related diagnosis were patients who had a laboratory-confirmed (RT-PCR assay) COVID-19.

Results: The study covered a total of 53 medical schemes' data. These accounted for 8,3 million lives. The analysis included a total of 3,437 admissions that had a viral pneumonia primary admissions ICD-10 code. Other viral pneumonia accounted for a significant proportion of the admissions; 82 percent. The second-highest group was viral pneumonia, unspecified, which accounted for 16 percent of admissions. Other types of viral pneumonia included respiratory syncytial virus pneumonia, adenoviral pneumonia and parainfluenza virus pneumonia, which accounted for the remaining 2 percent. The paid amount for other viral pneumonia compared to viral pneumonia, unspecified was not statistically significant, (R66 399 vs. R67 490; $p=0.8961$ ). The average inpatient days were also not significantly different ( 9.3 days vs. 8.7 days $p=0.1679$ ). Other viral pneumonia compared to viral pneumonia, unspecified were likely to have a COVID-19 confirmed secondary ICD-10 diagnosis, 63 percent versus 44 percent, $p<0.001$. Significantly, less other viral pneumonia compared to viral pneumonia had a suspected COVID-19 secondary ICD-10 admissions diagnosis, 2 percent vs. 18 percent, p<0.001. Lastly, significantly more other viral pneumonia compared to viral pneumonia had essential primary hypertension COVID-19 secondary ICD-10 admissions diagnoses, 5 percent vs. 2 percent, $p<0.001$

Conclusion: The study found that mainly the other viral pneumonia and the viral pneumonia, unspecified primary ICD-10 diagnoses were more likely to be admitted into hospitals. The study found that other viral pneumonia had a higher likelihood than viral pneumonia, unspecified to have a COVID-19 secondary ICD-10 admission diagnosis and were more likely to be associated with a COVID-19 admissions diagnosis. The World Health Organisation (WHO) warns that vaccines against pneumonia, such as pneumococcal vaccine and Haemophilus influenza type B (Hib) vaccine, do not protect patients against the new coronavirus. This study however provides evidence that the frequency of confirmed cases of other viral pneumonia, suggest that pneumonia and pneumococcal vaccines might be one of the considerations in the development of COVID-19 vaccines. The findings of this study are also consistent with new evidence that Hib and pneumococcal vaccination has significantly lowered severe morbidity and mortality in influenza epidemics and might have similar efficacy for mitigating coronavirus outbreaks.

Keywords

Viral pneumonia, COVID-19, Primary ICD-10 code, Secondary ICD-10 code, Diagnosis, Admissions, SARS-CoV-2

\section{Background}

Various studies have drawn similarities between respiratory conditions such as pneumonia and COVID-19 Respiratory Disease (e.g., viral pneumonia J12.8) or signs or symptoms of respiratory disease (e.g., shortness of breath R06.0, cough R05) a respiratory disease (e.g., viral pneumonia J12.8) are the most notable symptoms that are associated with COVID-19. A recent study by Willie and Motsepe found that dyspnoea is one of the symptoms found on admissions into hospitals [1]. Dyspnoea which is also known as shortness
*Corresponding author: Michael Mncedisi Willie, Policy Research and Monitoring, Council for Medical Schemes, Pretoria, South Africa

Accepted: July 15, 2021

Published online: July 17, 2021

Citation: Willie MM (2021) Descriptive Analysis of Factors Associated with COVID-19 (U07.1, U07.2), Viral Pneumonia (J12.8 and J12.9) and other Types of Admission Diagnosis. J Healthcare 4(1):64-69 
of breath or breath lessness is a subjective awareness of the sensation of uncomfortable breathing.

A study by Chen, et al. clarified the epidemiological and clinical characteristics of 2019-nCoV pneumonia and found that of the 99 patients with 2019-nCoV pneumonia [2], the average age of the patients was older than 50 years at 55.5 years (SD 13.1). Further more, the study found that patients with clinical manifestations of fever were at 83 percent, a cough was at 82 percent, shortness of breath was at 31 percent, muscle ache was at 11 percent, confusion was at 9 percent, a headache was at 8 percent, a sore throat was at 5 percent, rhinorrhoea was at 4 percent, chest pain was at 2 percent, diarrhoea was at 2 percent, and nausea and vomiting was at 1 percent.

A study by Atere, et al. showed that a dry cough, chest pain and dyspnoea were prominent in patients with lower respiratory tract symptoms on admission [3]. Other types of diagnosis for admission in a study by Willie and Motsepe included unspecified acute lower respiratory infection, other viral pneumonia, Adult respiratory distress syndrome, Bronchopneumonia, unspecified - Gastroenteritis, and colitis of unspecified origin [1].

\section{Introduction}

The COVID-19 pandemic has led to an unprecedented surge in hospitalised patients with viral pneumonia [4]. COVID-19 usually presents as an acute viral respiratory tract infection and many differential diagnoses related to common viral pneumonia should be considered [5]. Viral Pneumonia is also believed to be the cause of half of all cases of pneumonia [6]. Previous studies that have linked pneumonia with corona type viruses date back almost a decade. A study by Ruuskanen included Coronavirus types 229E, OC43, NL63, HKU1, and SARS as forming part of at least 26 viruses associated with community-acquired pneumonia (viral pneumonia) [6]. Both the COVID-19 and viral pneumonia use upper-respiratory specimens to test for viruses [6-9].

There is also a body of literature on the use of lower respiratory tract (sputum, tracheal aspirate, BAL) specimens to test for COVID-19 [10,11]. COVID-19 has also been detected in faeces, blood, urine, and breast milk, although the transmissibility of the virus from these fluids is unknown [12-14]. Recent studies have confirmed the presence of SARSCoV-2 nucleic acids in feces of Coronavirus disease 2019 (COVID-19) patients using RT-PCR tests $[8,15]$. A rapid review conducted by Jones, et al. found that SARS-CoV-2 RNA can be detected in faeces from some asymptomatic, mildly - and presymptomatic individuals [14]. Another study by Szymczak, et al. found that SARS-CoV-2 can be detected in the stool of some patients for longer than 4 weeks, suggesting that a stool may hold utility as an additional source for diagnosis [16]. This evidences various from other forms of testing methods for the presence and prevalence of the virus.

\section{The average length of stay}

The average length of stay in hospital of patients with COVID-19 varies by country and setting, with some countries reporting a longer length of stay (LoS) than others. A systematic review and data synthesis on the average length of stay revealed that a median LoS ranged from 4 to 53 days within China, and 4 to 21 days outside of China, across 45 studies [17]. The average length of stay is also a function of severity, comorbidities, and demographic characteristic such as age. A study describing the characteristics of COVID-19 patients in hospitals found that the time from onset to hospitalization decreased with age, from 7.3 days in the 20 to 65 year olds to 4.5 days in the $>80$ year olds $(p<0.0001)$ [18]. Other studies found that the length of stay increased with age. Anderson, et al. [19] found that 41 percent of COVID-19 adults aged $\geq 18$ years with severe COVID-19 that were consecutively hospitalised from the emergency department (ED) had a LOS of $\geq 9$ days.

\section{Admitting facilities}

Pneumonia is among the most common medical reasons for admissions. The CMS reported that 69 per 10000 beneficiaries were admitted in 2018 and this accounted for most medical inpatient admission type [20]. The average total expenditure during that period was R28 000 . The average length of stay was 4 days, which is slightly lower than that reported in the US of 5 days [21]. A study by Guo, et al. analysed clinical characteristics and predicted mortality risks among patients with viral pneumonia. The study found that the mean length of hospital stay in viral pneumonia patients was 20.72 (SD 20.25) days [22].

In a prospective study by Wong, et al. for patients in ICU, the most common reasons for admission were neuromuscular weakness, pneumonia, multiple traumas, and septic shock, in that order [23]. A study by Willie and Motsepe investigated diagnoses associated with COVID-19 (U07.1), Pneumonia unspecified (J18.9) and other types of admissions, on a restricted medical scheme case study in South Africa [1]. The study found that patients admitted for COVID-19 were chiefly admitted in the "other" type of admitting categories, compared to those admitted in the general ward, high care, and ICU [1]. The authors found that the admission rates were 55 percent, 29 percent, 14 percent and 2 percent, respectively. 'J18.9 Pneumonia, unspecified organism' admissions were chiefly in the general ward, compared to high care, ICU, and other types of admission [1].

\section{Differentiating between COVID-19 and viral pneumonia}

The literature depicts a link relationship between viral pneumonia and COVID-19. A study by Elmokadem, Bayoumi, Abo-Hedibah, et al. cited viral pneumonia as one of the commonest causes of the ground-glass opacities (GGO) that can simulate COVID-19 [24]. Other studies however differentiated between COVID-19 and Non-COVID-19 Viral Pneumonia. A study by Bai, Hsieh, Xiong, et al. revealed the ability of radiologists from China in differentiating COVID-19 from non-COVID-19 viral pneumonia, with an accuracy as high as 83 percent [25]. The authors concluded that radiologists in China and in the United States distinguished the coronavirus disease 2019 from viral pneumonia at chest CT with moderate to high accuracy. 


\section{Objectives}

The primary objective of this study was to study the characteristics of medical scheme patients with viral pneumonia, as a primary ICD-10 hospital admission diagnosis, as well as its association with ICD-10 secondary hospital admission diagnosis.

\section{Method}

The study design was a descriptive analysis of medical schemes admissions data that occurred in 2020. The review period was claims received between March to December 2020. The inclusion criteria were patient's with viral pneumonia primary ICD-10 code. Patients with a secondary ICD-10 code that had a COVID-19 related diagnosis had a laboratory-confirmed (RT- PCR assay) COVID-19. Inclusion criteria for COVID-19 admissions were patients who had viral pneumonia as a primary ICD-10 admission diagnosis. The following primary ICD-10 codes were used to identify the patients who were included in the analysis:

\section{J12.0: Adenoviral pneumonia}

\section{J12.8: Other viral pneumonia}

\section{J12.2: Parainfluenza virus pneumonia}

\section{J12.1: Respiratory syncytial virus pneumonia}

\section{J12.9: Viral pneumonia, unspecified}

A secondary ICD-10 diagnosis was classified into two groups, mainly a COVID-19 diagnosis and other types of diagnosis. COVID-19 admissions secondary ICD-10 were patients that had a laboratory-confirmed (RT- PCR assay) COVID-19. The World Health Organization (WHO) defines ICD-10 codes 'U07.1' and 'U07.2' as follows [9,26-28]: An emergency ICD-10 code of 'U07.1 COVID-19, virus identified is assigned to a diagnosis of COVID-19 confirmed by laboratory testing. An emergency ICD-10 code of 'U07.2 COVID-19, virus not identified is assigned to a clinical or epidemiological diagnosis of COVID-19 where laboratory confirmation is reported as inconclusive or not available. Both U07.1 and U07.2 may be used for mortality coding (cause of death).

\section{Statistical Analysis}

Data was processed using both SAS 9.4 and Stata statistical packages. The results were expressed as means and SDs or as the median and interquartile range (IQRs), as appropriate. The Student T-test was used to assess group differences for continuous variables and the Chi-square- test was used to assess group differences in categorical variables. $p$ values $<0.05$ were considered statistically significant.

\section{Results}

\section{Study population}

The study covered a total of 53 medical schemes' data. These accounted for 8,3 million lives. The analysis included a total of 3,437 admissions that had a viral pneumonia primary admissions ICD-10 code. Other viral pneumonia accounted for a significant proportion of the admissions; 82 percent, the second-highest group was viral pneumonia, unspecified, which accounted for 16 percent of admissions. Other types of viral pneumonia included respiratory syncytial virus pneumonia, adenoviral pneumonia and parainfluenza virus pneumonia, which accounted for the remaining 2 percent (Figure 1).

Table 1 below shows admissions comparison analysis between the primary and the secondary ICD-10 admission diagnosis. The results showed that other viral pneumonia compared to viral pneumonia, unspecified were likely to have a COVID-19 confirmed secondary ICD-10 diagnosis, 63 percent versus 44 percent, $p<0.001$. Significantly less Other viral pneumonia compared to viral pneumonia had a suspected COVID-19 secondary ICD-10 admissions diagnosis, 2 percent vs. 18 percent, $p<0.001$. Lastly, significantly more other viral pneumonia compared to viral pneumonia had essential primary hypertension COVID-19 secondary ICD-10 admissions diagnosis, 5 percent vs. 2 percent, $p<0.001$. The paid amount for other viral pneumonia compared to Viral pneumonia, unspecified was not statistically significant, R66 399: 95\% Cl (56 R126-R76 672) vs. R67 490: 95\% Cl (R54 700$R 80281) ; p=0.8961$. The average inpatient days were also not significantly different 9.3: $95 \% \mathrm{Cl}(8.8-9.8)$ days vs. 8.7: $95 \% \mathrm{Cl}(8.1-9.4)$ days $\mathrm{p}=0.1679$.

Other types of viral pneumonia were excluded, as these accounted for only 2 percent of admissions. Figure 2 below depicts a Box and Whisker plot of inpatient days, for both primary and secondary ICD-10 admission diagnosis. The plot depicts a few adenoviral pneumonia cases that are related to COVID-19 suspected cases. Respiratory syncytial pneumonia virus cases were related to other types of diagnosis rather than those that were COVID-19 related. Figures 3 and Figures 4 below depict the average amount paid and inpatient days per hospitalisation per admitting facility. The average paid

Table 1: Comparison analysis between primary and secondary ICD10 admission diagnosis.

\begin{tabular}{|c|c|c|c|c|}
\hline $\begin{array}{l}\text { Primary ICD-10 } \\
\text { Admission } \\
\text { Diagnosis }\end{array}$ & $\begin{array}{l}\text { Secondary ICD- } \\
10 \text { Admission } \\
\text { Diagnosis }\end{array}$ & Count & Total & $\%$ \\
\hline \multirow[t]{2}{*}{$\begin{array}{l}\text { Adenoviral } \\
\text { pneumonia }\end{array}$} & Covid-19 Suspected & 7 & \multirow[t]{2}{*}{9} & $78 \%$ \\
\hline & Other & 2 & & $22 \%$ \\
\hline \multirow[t]{3}{*}{$\begin{array}{l}\text { Other viral } \\
\text { pneumonia }\end{array}$} & Covid-19 Confirmed & 1776 & \multirow{3}{*}{2824} & $63 \%$ \\
\hline & Covid-19 Suspected & 69 & & $2 \%$ \\
\hline & Other & 979 & & $35 \%$ \\
\hline \multirow[t]{3}{*}{$\begin{array}{l}\text { Parainfluenza virus } \\
\text { pneumonia }\end{array}$} & Covid-19 Confirmed & 1 & \multirow{3}{*}{5} & $20 \%$ \\
\hline & Covid-19 Suspected & 3 & & $60 \%$ \\
\hline & Other & 1 & & $20 \%$ \\
\hline \multirow[t]{3}{*}{$\begin{array}{l}\text { Respiratory } \\
\text { syncytial virus }\end{array}$} & Covid-19 Confirmed & 3 & \multirow{3}{*}{36} & $8 \%$ \\
\hline & Covid-19 Suspected & 22 & & $61 \%$ \\
\hline & Other & 11 & & $31 \%$ \\
\hline \multirow[t]{3}{*}{$\begin{array}{l}\text { Viral pneumonia, } \\
\text { unspecified }\end{array}$} & Covid-19 Confirmed & 258 & \multirow{3}{*}{563} & $46 \%$ \\
\hline & Covid-19 Suspected & 99 & & $18 \%$ \\
\hline & Other & 206 & & $37 \%$ \\
\hline
\end{tabular}


Citation: Willie MM (2021) Descriptive Analysis of Factors Associated with COVID-19 (U07.1, U07.2), Viral Pneumonia (J12.8 and J12.9) and other Types of Admission Diagnosis. J Healthcare 4(1):64-69

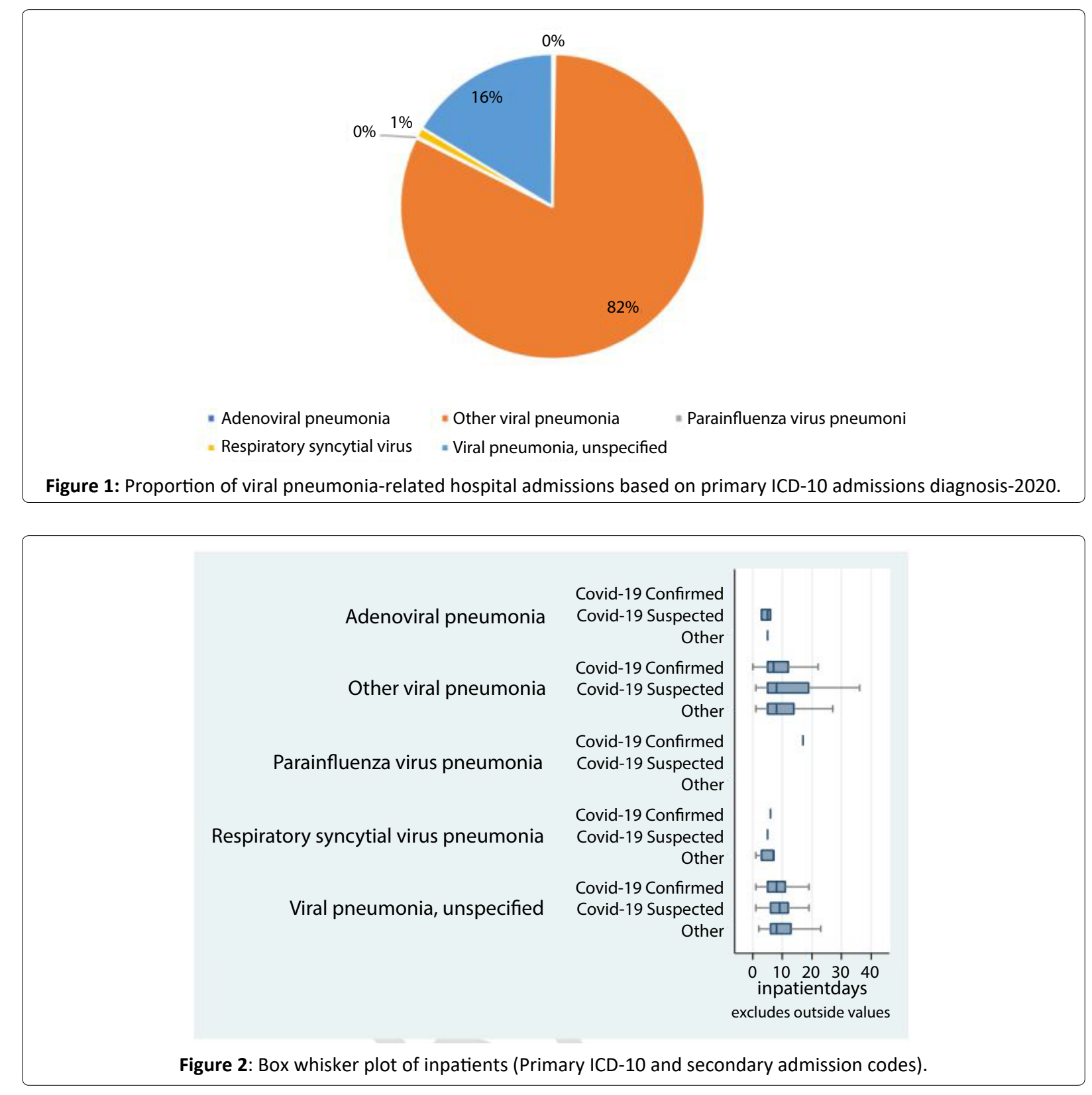

Figure 2: Box whisker plot of inpatients (Primary ICD-10 and secondary admission codes).

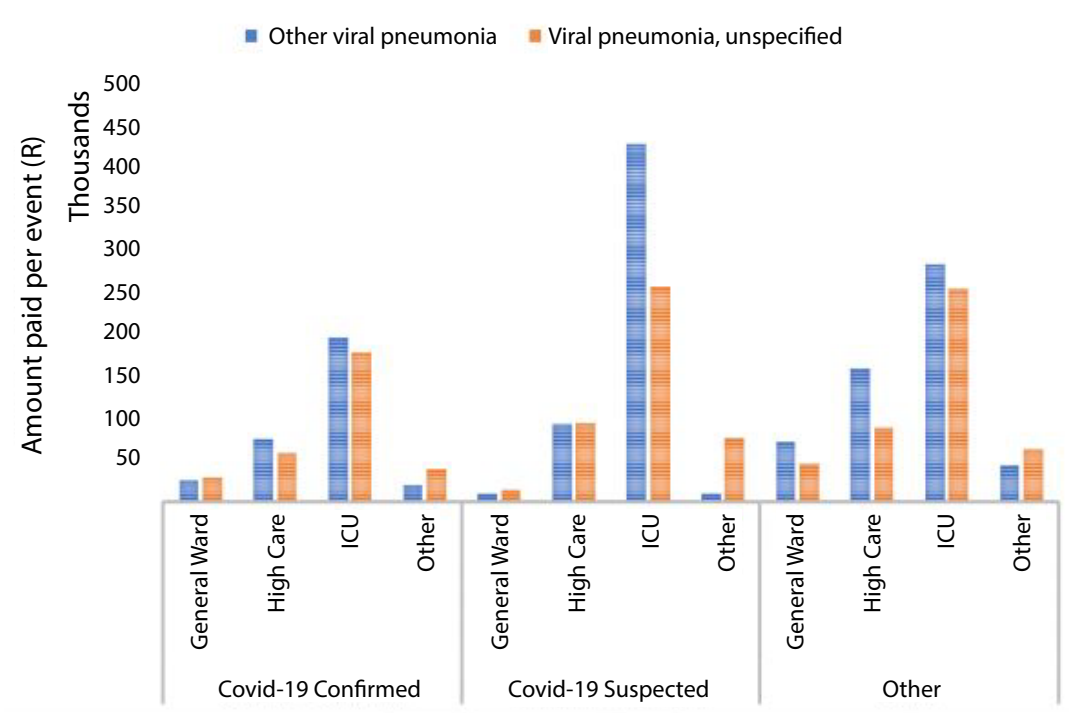

Figure 3: Claim amount paid per hospitalisation per diagnosis (Primary and Secondary ICD-10 diagnosis and admitting facility). 
Citation: Willie MM (2021) Descriptive Analysis of Factors Associated with COVID-19 (U07.1, U07.2), Viral Pneumonia (J12.8 and J12.9) and other Types of Admission Diagnosis. J Healthcare 4(1):64-69

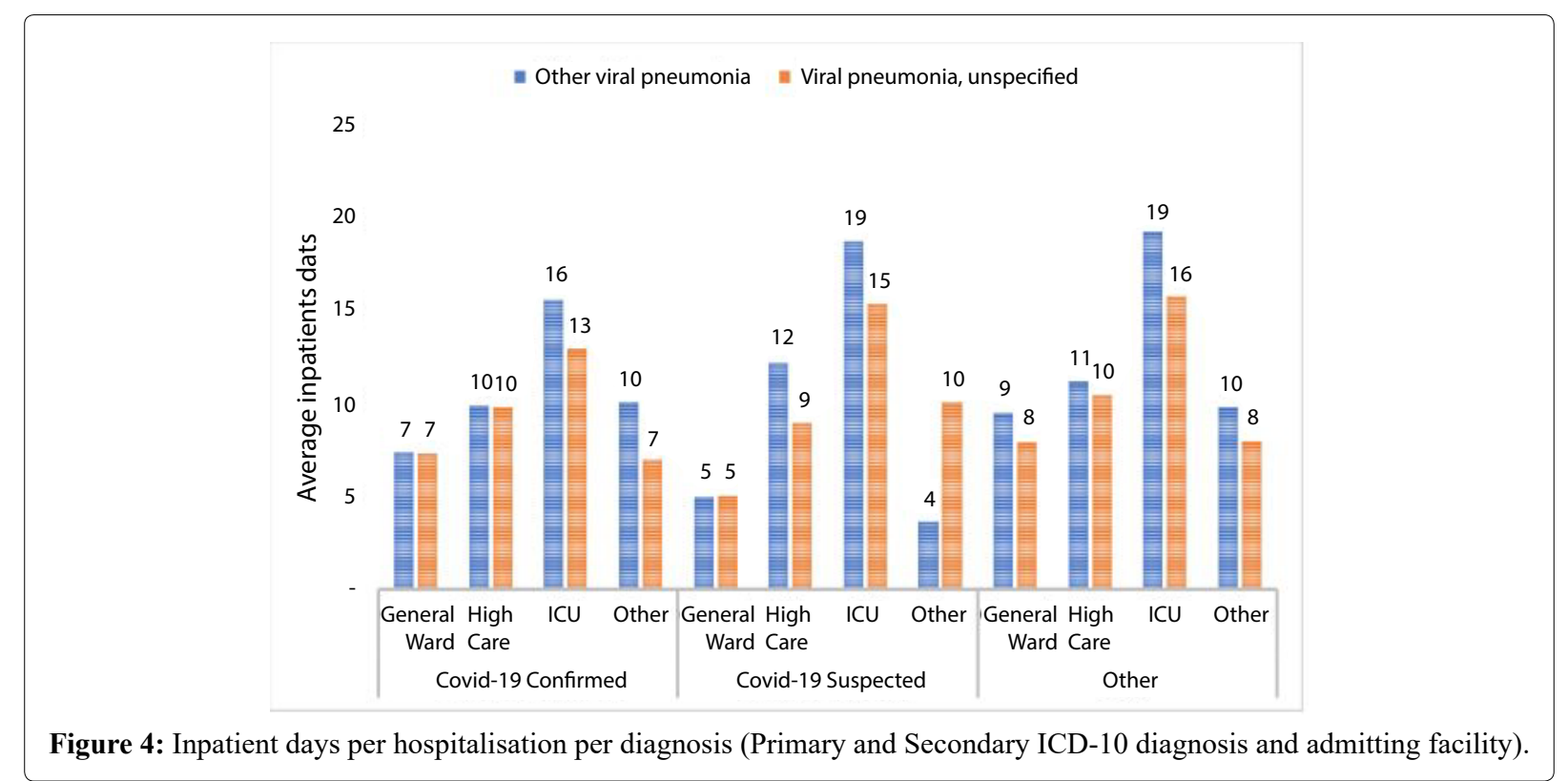

amount per event was consistently higher for other viral pneumonia compared to viral pneumonia, unspecified that had a COVID-19 related secondary diagnosis. However, this was reversed in other types of diagnosis. Primary ICD-10 diagnoses that had a COVID-19 secondary diagnosis should be interpreted with caution, due to the small number of observations in this group.

\section{Discussion}

The study found that mainly the other viral pneumonia and viral pneumonia, unspecified primary ICD-10 diagnosis were more likely to be admitted into hospitals. In the main, other viral pneumonia accounted for a significant proportion of the admissions at 82 percent. Various studies found that viral pneumonia was the most common indication for hospital admission and over 80 percent of hospitalised patients were cared for in general medical wards $[4,29,30]$. This study found that other viral pneumonia had a higher likelihood than viral pneumonia, unspecified to have a COVID-19 secondary ICD10 admission diagnosis. This finding is consistent with the literature, in that COVID-19 usually presents as an acute viral respiratory tract infection and that many different diagnoses related to common viral pneumonia should be considered [5]. A study by Ruuskanen included Coronavirus types 229E, OC43, NL63, HKU1, and SARS as one of at least 26 viruses associated with community-acquired pneumonia (viral pneumonia) [6]. This study is one of the very few studies that contrast the differentiation between other viral pneumonia and COVID-19. Further more, the study shows that other viral pneumonia compared to viral pneumonia, unspecified were more likely to have a COVID-19 confirmed secondary ICD-10 diagnosis, 63 percent versus 44 percent. A study by Bai, Hsieh, Xiong, et al. revealed the ability of radiologists from China in differentiating COVID-19 from non-COVID-19 viral pneumonia with an accuracy as high as 83 percent [25]. The expenditure per hospital event for other viral pneumonia was higher than that of viral pneumonia, unspecified, based on primary ICD10 diagnosis. However, these were not statistically significant.
A similar finding was noted on the observed length of stay showing which were more likely to be admitted to hospitals.

This study found that patients with other viral pneumonia primary diagnosis which was linked to a COVID-19 secondary ICD-10 diagnosis showed that average inpatients days in an ICU setting were 16 and 13 days, respectively. Willie and Motsepe found that COVID-19 ICU inpatients stayed longer than 'J18.9 Pneumonia, unspecified organism' diagnosis, with median inpatient days of 13 and 6 days, respectively. A similar phenomenon was also observed in high care admissions, where the median inpatient days was 9 days vs. 7 days, respectively [1]. A study by Guo, et al. analysed clinical characteristics and predicted mortality risk among patients with viral pneumonia. The study found that the mean length of hospital stay in viral pneumonia patients was 20.72 (SD 20.25) days, which is higher than those in this study [22].

\section{Conclusion}

The study found that mainly the other viral pneumonia and the viral pneumonia, unspecified primary ICD-10 diagnosis were more likely to be admitted to hospitals. The study found that other viral pneumonia had a higher likelihood than viral pneumonia, unspecified to have a COVID-19 secondary ICD-10 admission diagnosis; which is more likely to be associated with a COVID-19 admissions diagnosis. The World Health Organisation (WHO) warns that vaccines against pneumonia, such as pneumococcal vaccine and Haemophilus influenza type $B$ (Hib) vaccine, do not protect against the new coronavirus. This study however provides evidence that the frequency of confirmed cases of other viral pneumonia, such as pneumonia and pneumococcal vaccines might be one of the considerations in the development of COVID-19 vaccines. The findings of this study are also consistent with new evidence that Hib and pneumococcal vaccination has significantly lowered severe morbidity and mortality in influenza epidemics and might have similar efficacy for mitigating coronavirus outbreaks. 


\section{Competing Interests}

The author has declared that no competing interest exists.

\section{Authors Contributions}

The author drafted and proofread the article.

\section{References}

1. Willie MM, Motsepe A (2021) Diagnosis associated with COVID-19 (U07.1), Pneumonia- Unspecified (J18.9) and other types of admissions: A restricted medical scheme case studySouth Africa. E3 Journal of Medical Research 8: 001-016.

2. Chen Y, Liangjun Chen, Qiaoling Deng, et al. (2020) The presence of SARS-CoV-2 RNA in the feces of COVID-19 patients. J Med Virol 92: 833-840.

3. Atere M, Singh S, Arora K, et al. (2020) COVID-19: The case of three patients with the same diagnosis but different clinical and laboratory features. Case Rep Med 2020: 9185041.

4. George PM, Barratt SL, Condliffe R, et al. (2020) Respiratory followup of patients with COVID-19 pneumonia. Thorax 75: 1009-1016.

5. Chia $\mathrm{Wu} Y \mathrm{Y}$, Chen Ching Sunga, Chan Yu Jiuna (2020) The outbreak of COVID-19: An overview. J Chin Med Assoc 83: 217-220.

6. Ruuskanen O, Lahti E, Jennings LC, et al. (2011) Viral pneumonia. Lancet 377: 1264-1275.

7. Palmas G, Maria Moriondo, Sandra Trapani, et al. (2020) Nasal swab as preferred clinical specimen for COVID-19 testing in children. Pediatr Infect Dis J 39: e267-e270.

8. Byrne RL, Grant A Kay, Konstantina Kontogianni, et al. (2020) Saliva alternative to upper respiratory swabs for SARS-CoV-2 diagnosis. Emerging Infectious Diseases 26: 2769-2770.

9. WHO (2020b) Media statement: Knowing the risks for COVID-19. Geneva.

10. Zheng S, Fan J, Yu F, et al. (2020) Viral load dynamics and disease severity in patients infected with SARS-CoV-2 in Zhejiang province, China, January-March 2020: Retrospective cohort study. BMJ 369: m1443.

11. Martinez RM (2020) Clinical Samples for SARS-CoV-2 Detection: Review of the early literature. Clinical Microbiology Newsletter 42: 121-127.

12. Murphy K (2020) SARS CoV-2 detection from upper and lower respiratory tract specimens: Diagnostic and infection control implications. Chest 158: 1804-1805.

13. Chambers $C$, Krogstad P, Bertrand K, et al. (2020) Evaluation for SARS-CoV-2 in breast milk from 18 infected women. JAMA 324: $1347-1348$.

14. Jones DL, Marcos Quintela Baluja, David W Graham, et al. (2020) Shedding of SARS-CoV-2 in feces and urine and its potential role in person-to-person transmission and the environment-based spread of COVID-19. Sci Total Environ 749: 141364.
15. Brogna B, Carlo Brogna, Mauro Petrilloet al. (2021) SARS-CoV-2 detection in fecal sample from a patient with typical findings of COVID-19 pneumonia on CT but negative to multiple SARSCoV-2 RT-PCR tests on oropharyngeal and nasopharyngeal swab samples. Medicina (Kaunas) 57: 290.

16. Szymczak WA, Yitzchak Goldstein D, Erika Orner P, et al. (2020) Utility of stool PCR for the diagnosis of COVID-19: Comparison of two commercial platforms. J Clin Microbiol 58: e01369-20.

17. Rees EM, Nightingale ES, Jafari Y, et al. (2020) COVID-19 length of hospital stay: A systematic review and data synthesis. BMC Med 270.

18. Boelle PY, Tristan Delory, Xavier Maynadier, et al. (2020) Trajectories of hospitalization in COVID-19 patients: An observational study in France. J Clin Med 9: 3148.

19. Anderson MR, Bach PB, Baldwin MR (2021) Hospital length of stay for patients with severe COVID-19: Implications for remdesivir's value. Pharmacoecon Open 5: 129-131.

20. Council for Medical Schemes CMS (2019) Annual report 201819. Pretoria: South Africa.

21. Agency for healthcare research and quality (2021) Average length of stay. Quality of care-healthcare cost and utilization project nationwide.

22. Guo L, Wei D, Zhang X, et al. (2019) Clinical features predicting mortality risk in patients with viral pneumonia: The MuLBSTA score. Front Microbiol 10: 2752.

23. Wong DT, Gomez M, McGuire GP, et al. (1999) Utilization of intensive care unit days in a canadian medical-surgical intensive care unit. Crit Care Med 27: 1319-1324.

24. Elmokadem AH, Bayoumi D, Abo Hedibah SA, et al. (2021) Diagnostic performance of chest CT in differentiating COVID-19 from other causes of ground-glass opacities. Egypt J Radiol Nucl Med 52: 12.

25. Bai HX, Hsieh B, Xiong Z, et al. (2020) Performance of radiologists in differentiating COVID-19 from viral pneumonia on chest CT. Radiology 296: E46-E54.

26. WHO (2020a) Laboratory testing strategy recommendations for COVID-19: Interim guidance.

27. WHO (2020c) International guidelines for certification and classification (coding) of COVID-19 as cause of death. Geneva.

28. WHO (2020d) Emergency use ICD codes for COVID-disease outbreak. Geneva.

29. Najafi S, Sandrock C (2017) Hospitalized patients with acute pneumonia. Hosp Med Clin 6: 456-469.

30. Choi SH, Hong SB, Ko GB, et al. (2012) Viral infection in patients with severe pneumonia requiring intensive care unit admission. Am J Respir Crit Care Med 186: 325-332.

DOI: $10.36959 / 569 / 465$

Copyright: (C 2021 Rachdi I. This is an open-access article distributed under the terms of the Creative Commons Attribution License, which permits unrestricted use, distribution, and reproduction in any medium, provided the original author and source are credited. 